\title{
Abakterielle Cystitiden: erfolgreich reparieren, langfristig stabilisieren
}

— Seit längerem ist der Wirkstoff NatriumPentosanpolysulfat (PPS) zur Behandlung der Symptome einer Interstitiellen Zystitis (IC) etabliert. Die Europäische Urologische Gesellschaft führt in ihren Richtlinien PPS als Standardtherapie zur oralen Behandlung einer IC auf. Belegt ist die Wirksamkeit und Verträglichkeit von PPS durch klinische Studien. Mehrere Untersuchungen zeigen, dass die IC durch eine Dysfunktion der aus Glycosaminoglycanen bestehenden Schicht der Blasenepithelzellen (GAG-Schicht) verursacht wird. Dadurch können Schadstoffe und Kaliumionen in die umgebende Blasenwand gelangen, was zu einer Entzündung des Gewebes, zur Irritation und Sensibilisierung der sensorischen Nerven führt. Der vorübergehende Ersatz der GAG-Schicht mit PPS verschafft dem Urothel günstigere Bedingungen, sich zu regenerieren und eine neue schützende Schleimschicht aufzubauen. Genau dies leistet das Medizinprodukt cyst-u-ron ${ }^{\circledR}$. Der bewährte Wirkstoff PPS ist unter diesem Namen neu als gebrauchsfertige Blaseninstillationslösung (300 mg PPS) in Apotheken erhältlich.

Nach Informationen von bene pharmaChem, Geretsried

\section{Temsirolimus im Praxistest: Studie erweitert}

\begin{abstract}
- Das Patientenregister STAR-TOR von Wyeth Pharma nimmt seit Dezember 2009 auch Patienten mit einem rezidivierenden und/oder refraktären Mantelzell-Lymphom $(\mathrm{MCL})$ auf, die eine Therapie mit Temsirolimus (Torisel ${ }^{\circledR}$ ) erhalten. Ziel der nicht interventionellen Studie STAR-TOR ist es, die Wirksamkeit, Sicherheit und Verträglichkeit der Therapie mit Temsirolimus unter Praxisbedingungen zu beobachten, um diese mit den Ergebnissen aus klinischen Studien zu vergleichen. STAR-TOR wurde bereits im Februar 2008 für Hochrisikopatienten mit fortgeschrittenem Nierenzellkarzinom gestartet. Mit der Erweiterung sollen nun auch die Daten der zweiten zugelassenen Indikation von Temsirolimus in der praktischen Anwendung untersucht
\end{abstract}

werden. Um repräsentative Daten zu erhalten, ist eine große Patientenbeteiligung notwendig. Bisher wurden insgesamt 312 Patienten aus 92 Zentren rekrutiert. Die Auswertung zum Nierenzellkarzinom bestätigte bisher die guten Ergebnisse aus der klinischen Studie.

Ärzte, die Patienten in das Patientenregister aufnehmen lassen möchten, können sich an das Medizinische Informations- und Kundenservicecenter von Wyeth Pharma wenden, unter der Tel.: 01802783993 (6ct./Anruf) oder per Mail an:mik_email@ wyeth.com.

\section{Nach Informationen von}

Wyeth, Münster

\section{Praxis-Website in 15 Minuten}

— Für Ärzte, die den Weg ins Netz bisher noch nicht beschritten haben, gibt es seit kurzem „docvadis“ (www.docvadis.de) - einen neuen Service der Firma MSD. Damit können Ärzte in einer Viertelstunde ihre eigene Website gestalten. Nur fünf Schritte braucht es dazu: Zunächst wird ein Layout ausgewählt, dann werden Informationen zur Tätigkeit und zur Praxis abgefragt. Schließlich gilt es, die Sprechzeiten anzu- geben und die Adresse des Auftritts zu personalisieren. Auch für die Füllung der Internetseite ist gesorgt: In der „Dokumentenbibliothek" stehen Fachartikel, Dokumente, Videos, Links und interaktive Inhalte zu vielen Krankheiten zur Verfügung, um sie in die Praxis-Website einzubinden.

Nach Informationen von

MSD Sharpe \& Dohme, Haar

\section{Fünf Jahre Astellas}

Der Arzneimittelhersteller Astellas feiert dieses Jahr fünfjähriges Jubiläum. 2005 entstand das Unternehmen aus einer Fusion der beiden japanischen Firmen Fujisawa und Yamanouchi. Anlässlich seines fünften Geburtstags betont Astellas, dass es neben dem weiteren Ausbau der therapeutischen Schwerpunktgebiete Urologie und Transplantation sein Betätigungsfeld zukünftig um den Therapiebereich Onkologie erweitern werde. „Die Onkologie bietet für Astellas große Chancen und wir sind sehr zuversichtlich, dass wir auch in diesem neuen Geschäftsbereich den Erfolg, den wir in den letzten fünf Jahren in anderen Indikationsgebieten gehabt haben, wiederholen werden“, erläutert Dr. Ulrich Eggert, Geschäftsführer der Astellas Pharma GmbH.

Nach Informationen von

Astellas, München

\section{Jahre Pohl-Boskamp}

Seinen Anfang nahm die Firmenhistorie von Pohl-Boskamp in zwei Apotheken in Berlin und Danzig, die Ende des 19. Jahrhunderts als erste im großen Maßstab Gelatine-Kapseln für medizinische Zwecke produzierten. 1885 ließ sich die damalige Firma G. Pohl das „Verfahren zur Herstellung von Dünndarmkapseln“ mittels Keratin patentieren. Die Weiterentwicklung dieser Technik bis hin zur modernen magensaftresistenten Weichkapsel prägte die Geschichte des Unternehmens, das dieses Jahr seinen 175 . Geburtstag feiert.

Heute umfasst das Produktportfolio von Pohl-Boskamp 40 verschiedene Arzneimittel und Medizinprodukte. Eine Ausweitung des Sortiments auf die Urologie erfolgte 2003 mit Gepan ${ }^{\circledR}$ instill, einer ChondroitinsulfatLösung zur kausalen Behandlung chronischer Blasenentzündungen.

Nach Informationen von

Pohl-Boskamp, Hohenlockstedt 